Sociologie et sociétés

SOCIOLOGIE

ETSOCIÉTÉS

\title{
Vers une science du texte social
}

\section{Le (re)-commencement de la sociologie marxiste}

\section{Towards a Science of Social Context}

Hacia una ciencia del texto social

\section{Émilio DE IPOLA}

Volume 2, numéro 1, mai 1970

URI : https://id.erudit.org/iderudit/001360ar

DOI : https://doi.org/10.7202/001360ar

Aller au sommaire du numéro

\section{Éditeur(s)}

Les Presses de l'Université de Montréal

\section{ISSN}

0038-030X (imprimé)

1492-1375 (numérique)

Découvrir la revue

\section{Citer cet article}

DE IPOLA, É. (1970). Vers une science du texte social : le (re)-commencement de la sociologie marxiste. Sociologie et sociétés, 2(1), 123-144.

https://doi.org/10.7202/001360ar
Résumé de l'article

À partir d'une lecture critique de Métier de sociologue par P. Bourdieu, J.-C. Chamboredon et J.-C. Passeron, et de Pouvoir politique et classes sociales par N. Poulantzas, l'auteur analyse les rapports complexes entre la pratique sociologique, l'épistémologie marxiste et le matérialisme historique, ce dernier étant conçu comme science des modes de production et des formations sociales. Il s'agit de montrer la complémentarité nécessaire entre l'épistémologie et la réflexion théorique en vue de l'établissement de concepts de base visant à définir l'objet propre de la sociologie et à lui conférer ainsi son droit à l'existence théorique. 


\title{
Vers une science du texte social
}

\author{
le (re)-commencement de la sociologie marxiste *
}

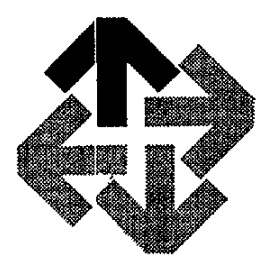

EMILIO DE IPOLA

$\mathrm{E}_{\mathrm{N}}$ SE POSANT comme théorie du texte social, la pratique sociologique amorce une démarche théorique qui implique une rupture décisive avec ses complicités traditionnelles ${ }^{1}$. Encore faut-il préciser que l'efficacité de ce geste a comme condition la mise en question radicale de toute herméneutique du social. Il est donc naturel qu'une telle tentative trouve dans l'espace théorique ouvert par la problématique marxiste les points de départ, les moyens conceptuels et, par conséquent, la possibilité même de son accomplissement. Car c'est dans le Capital que sont produits les instruments théoriques et méthodologiques indispensables à une telle entreprise : Marx y accomplit, en effet, dans un seul et même mouvement: théorique, d'une part la fondation du matérialisme historique (ou science de l'histoire des modes de production et des formations sociales), d'autre part la critique définitive de l'herméneutique comme idéologie de la lecture, ainsi que de toute lecture herméneutique des rapports sociaux. Bien entendu, ces deux démarches sont indissociables : la mise en place des concepts fondamentaux du matérialisme historique va de pair, en effet, avec la critique des présupposés philoso-

* A propos (et à partir) de : P. Bourdieu, J.-C. Chamboredon et J.-C. Passeron, le Métier de sociologue, Paris, Mouton-Bordas, 1968, liv. I; N. Poulantzas, Pouvoir politique et classes sociales, Paris, F. Maspero, 1968. Nous emploierons désormais les abréviations $M S$ et $P P C S$ pour indiquer les références à ces deux ouvrages.

1. Dans cette direction s'orientent les analyses présentes de Daniel Vidal, à propos du lien entre idéologie et rapports sociaux. 
phiques et théoriques de la formation idéologique dont la nouvelle science se détache, à savoir l'économie politique classique de Smith et de Ricardo. Mais cette critique est double : d'une part, Marx, lecteur des économistes classiques, transgresse le cercle herméneutique qui informe la pratique idéologique de la lecture ${ }^{2}$, d'autre part il opère la dé-construction de l'économie politique classique en montrant qu'elle pratique bel et bien une herméneutique du texte social. L'imbrication profonde de cette double critique apparait clairement dans ce texte exemplaire qu'est le chapitre XIX du livre I du Capital. Comme Althusser l'a montré, la lecture de l'économie classique par Marx ne se laisse enfermer ni dans les limites de la lecture littérale, ni dans celles de la lecture herméneutique. C'est par contre une lecture «symptomale », qui intervient dans la texture unique et actuelle du discours, en essayant d'y rendre compte de l'articulation significative du dit et du non-dit, des présences et des absences, et des effets spécifiques de cette articulation : condensations, déplacements, contradictions, ambivalences : symptômes ${ }^{3}$.

Mais la critique de l'herméneutique comme principe de lecture s'accompagne, dans la même analyse, d'une critique visant cette fois-ci la lecture d'un tout autre « texte * : celui des rapports de production capitaliste. Ainsi, si Ricardo a appris à lire, sous les « apparences » de l'échange, la substance de la valeur et sa mesure, sa problématique théorique, impuissante à produire le concept capital de « force de travail » et à saisir le caractère double du travail cristallisé dans la marchandise, se heurte à des contradictions insurmontables dès qu'il est question d'analyser cet objet énigmatique dont la lecture ricardienne tire justement son principe : le travail. On sait que la question-problème où se condensent les vues et les bévues de l'économie classique est celle de la détermination de la valeur du travail, «expression irrationnelle » dit Marx, dont, pourtant, la rationalité profonde doit pouvoir être montrée : «Déclarer que ces expressions irrationnelles sont pure licence poétique, c'est tout simplement une preuve de l'impuissance de l'analyse ${ }^{4}$.»

En fait, cette formule (valeur du travail) et le «problème \& qu'elle pose sont l'effet de l'incidence dans le texte même de l'économie politique traditionnelle des idéologies pratiques qui informent l'action des «agents-supports * de la production ${ }^{5}$; il s'agit, en l'occurrence, de ce que Marx appelle «les mystifications de la production capitaliste, toutes les illusions libérales et tous les faux-fuyants apologétiques de l'économie vulgaire ${ }^{6}>$.

Or, une lecture elle-même acritique, non symptomale, du chapitre XIX du Livre I, laisserait croire que Marx se limite simplement à dissiper ces illusions

2. Cf. L. Althusser, * Préface » à Lire le Capital I, Paris, Maspero, 1965.

3. Cf. ibid. et notre article : « Lectura y Politica. A propósito de Althusser *, Buenos Aires, éd. Galerna, 1970.

4. Capital, vol. II, p. 208. Toutes nos citations du Capital sont empruntées à la traduction des Editions sociales ( 8 vol.).

5. Par idéologies pratiques il faut entendre des formations complexes de montages de notions-représentations-images d'une part et de montages de comportements-conduitesattitudes-gestes d'autre part. L'ensemble fonctionne comme des normes pratiques qui gouvernent l'attitude et la prise de position concrète des hommes à l'égard des objets réels et des problèmes réels de leur existence sociale et individuelle, et de leur histoire * (L. Althusser, cité par M. Pécheux et E. Balibar, dans M. Fichant et M. Pécheux, Sur l'histoire des sciences, Paris, F. Maspero, 1969, p. 10, note).

6. Capital, vol. II, p. 211. 
idéologiques et à dévoiler ensuite le rapport réel, invisible pour l'idéologie, qui détermine la valeur de la force de travail et, par conséquent, le salaire. Mais à restreindre ainsi la portée de son analyse, on ignore le fait que, pour Marx, la grille idéologique, qui commande une certaine lecture du texte social par les agents de la production, fait partie, elle aussi, de ce texte; on oublie l'efficacité, non point apparente, mais bien réelle, de l'effet de reconnaissance-méconnaissance produit par cette lecture : "On comprend maintenant l'immense importance que possède dans la pratique ce changement de forme qui fait apparaître la rétribution de la force de travail comme salaire du travail, le prix de la force comme prix de sa fonction. $\gg$ (Capital, II, p. 211; c'est nous qui soulignons).

L'irrationnel trouve donc sa nécessité dans la logique même de la pratique capitaliste, s'il est vrai que la superstructure idéologique - aussi bien que la superstructure juridico-politique - est, pour Marx, condition d'existence et d'intelligibilité de la structure du mode de production capitaliste. Les formes phénoménales s'avèrent être ainsi des formes essentielles, ce qui revient à dire que le couple "phénomène vs essence » - invariant de toute herméneutique ne saurait nullement rendre compte de la démarche propre de l'analyse marxiste.

S'il en est ainsi, le renouvellement de la pratique sociologique est concerné par les effets théoriques de l'entreprise marxiste. Ces effets, on le sait, portent sur un double registre : a) celui de la nouvelle science, le matérialisme historique, science dont on peut bien dire qu'elle inaugure une nouvelle lecture de la pratique; b) celui de la discipline vouée traditionnellement à la réinscription idéologique du discours scientifique, à savoir la philosophie ?, qui devient après Marx matérialiste et dialectique, capable donc de se tenir à distance de l'idéologique et d'informer par là même une nouvelle pratique de la lecture de ce discours. En ce sens, l'intérêt qu'à notre avis possèdent les ouvrages de P. Bourdieu, J.-C. Chamboredon et J.-C. Passeron : le Métier de sociologue et de N. Poulantzas : Pouvoir politique et classes sociales réside en ceci que, chacun à leur niveau, ils développent un travail théorique permettant de penser rigoureusement les rapports complexes qui relient la pratique sociologique, d'une part à l'épistémologie matéraliste et, d'autre part, à la théorie de l'histoire. Autant dire que, dans la conjoncture théorique actuelle, ces travaux constituent des points de repère indispensables pour toute tentative visant à définir, sous une forme conceptuelle, le lieu théorique de la sociologie. C'est d'une lecture qui se veut non herméneutique et non littérale de ces textes que proviennent les remarques qui suivent.

\section{MATÉRIALISME DIALECTIQUE ET SOCIOLOGIE}

Nous entendons par matérialisme dialectique (ou philosophie marxiste) ${ }^{8}$ une discipline dont la « fonction majeure est de tracer une ligne de démarcation entre l'idéologique des idéologies d'une part, et le scientifique des sciences d'autre part ${ }^{\natural} \gg$. La pratique épistémologique est ainsi comprise comme une intervention

7. Dans la suite, nous employons comme des synonymes les termes de c philosophie », - épistémologie > et * matérialisme dialectique *.

8. Voir note 7.

9. L. Althusser, «Introduction », Cours de philosophie pour scientifiques (ronéotypé). 
spécifique dans la réalité indistincte, impure, où se réalise l'«impossible » conjonction de l'idéologique et de ce qui est par principe le non-idéologique radical : le discours scientifique. Cette intervention a donc un effet critique, car c'est en produisant constamment (au sens de rendre visible) la différence entre le scientifique et l'idéologique, qu'elle dénonce - et permet ainsi d'éviter — les obstacles épistémologiques qui menacent la production réglée des connaissances. Tracer des lignes de démarcation, produire et reproduire incessamment le clivage entre connaissance et méconnaissance, suppose en fait concevoir l'épistémologie comme une sorte de pratique politique, dont l'enjeu sont les sciences, et l'objectif la promotion du scientifique au sens strict. La pratique épistémologique implique donc une véritable «prise de parti » en faveur des sciences et contre les idéologies qui les assiègent.

Ce n'est pas tout. L'intervention épistémologique se rapproche aussi de la politique en ceci qu'elle s'exerce sur une réalité ayant une forme d'existence "conjoncturelle», à savoir les pratiques scientifiques. Par ces dernières, il faut entendre des formations théoriques complexes, caractérisées a) par la coexistence articulée du travail scientifique et du travail idéologique; $b$ ) par une dominance particulière, celle du travail scientifique, du moins dans les sciences « développées».

Or, la pratique sociologique constitue bien entendu une de ces formations à cette différence près que, en règle générale, la dominance est exercée à l'heure actuelle par l'idéologique. Qu'on ne se méprenne pourtant pas sur le sens et la portée de cette affirmation : nous sommes loin de contester à la sociologie son droit à l'existence théorique; encore moins proposons-nous bien au contraire, de mettre en question les efforts des chercheurs visant à donner à la sociologie des instruments conceptuels et méthodologiques rigoureux, c'est-à-dire scientifiques. Nous nous limitons à constater une réalité conjoncturelle, explicitement ou implicitement reconnue par ces mêmes chercheurs ${ }^{10}$, réalité dont on doit pouvoir mesurer les effets théoriques si on veut renverser le rapport des forces et faire passer le travail scientifique au rôle d'élément dominant dans la pratique sociologique.

C'est précisément cette réalité conjoncturelle qui réclame de toute urgence l'intervention épistémologique. $\grave{A}$ celle-ci revient en effet la tâche de repérer les coupures déjà produites par la recherche sociologique et de promouvoir la production des connaissances à travers la dénonciation des formations idéologiques dominantes. Autant dire qu'une intervention épistémologique efficace suppose nécessairement une intelligence approfondie de cette conjoncture théorique qui est la nôtre.

Or, c'est sans doute dans ces termes que le Métier de sociologue ${ }^{11}$ entend la surveillance épistémologique. L'épistémologie y est conçue en effet comme

10. Cf. par exemple A. Touraine : a Il faut ... admettre la contamination doctrinale de la majorité des études (sociologiques) publićes... $»$ (Sociologie de l'action, Paris, Seuil, 1965 , p. 114). Pour Touraine il s'agit simplement d'une situation de fait, susceptible d'être dépassée. D'autres sociologues la considèrent bel et bien comme une caractéristique constitutive et indépassable de la sociologie. Or, si l'idée d'une science absolument débarrassée d'idéologie relève d'une utopie positiviste, il reste toujours vrai qu'une science ne progresse que dans la mesure où elle réussit progressivement à saisir, puis à annuler, les attaques (pourtant toujours renouvelées) de l'idéologique.

11. Cf. $M S$, "Avant-propos 》. 
l'exercice rigoureux d'un double contrôle, syntaxique et sémantique, logique et conceptuel, portant sur l'ensemble des opérations qui définissent la pratique sociologique (à savoir, notamment, les opérations théoriques visant la construction de l'objet d'analyse et les opérations méthodologiques visant le contrôle des énoncés) et sur chacune des étapes du processus de production des connaissances sociologiçues (à savoir, notamment, l'étape d'investigation ou de recherche au sens fort du terme, et l'étape d'exposition ou de présentation des concepts sous forme d'un discours démonstratif). Il s'agit, selon les auteurs, de soumettre l'ensemble des procès de travail sociologique «à la polémique de la raison épistémologique pour définir et, s'il se peut, inculquer une attitude de vigilance qui trouve dans la connaissance adéquate de l'erreur et des mécanismes capables de l'engendrer un des moyens de la surmonter $\gg(M S$, p. 9).

«Sur-moi » de la pratique sociologique, l'épistémologie est aussi, d'après le Métier de sociologue, politique : elle agit «ici et maintenant», sur le moment actuel, sur cette conjoncture sociologique qui est la nôtre, et exige par conséquent une connaissance poussée des formations théoriques et des obstacles idéologiques dominants, ainsi que des lignes de force et des tendances qui s'y dessinent. En ce sens, les analyses de l'ouvrage sont une excellente illustration des effets critiques et positifs que l'on peut atteindre d'une intervention épistémologique ainsi informée. Il convient pourtant de distinguer, dans ces analyses, la démarche proprement critique et les propositions positives visant à esquisser une «stratégie théorique » destinée à surmonter les difficultés de la sociologie actuelle. Les raisons d'une telle distinction apparaîtront bientôt.

La démarche critique de Bourdieu, Chamboredon et Passeron tire son efficacité d'être basée, comme nous l'avons indiqué, sur une analyse serrée visant à mettre en lumière, dans la pratique sociologique, les «mécanismes capables d'engendrer l'erreur », autrement dit les formes de pénétration de l'idéologie au sein même de cette pratique. Analyse qui rompt heureusement avec une vieille tradition épistémologique - dont le poids se fait encore sentir en sociologie qui tend à concevoir l'erreur, selon les mots de G. Canguilhem, «comme un accident regrettable, une maladresse qu'un peu moins de précipitation ou de prévention nous eût épargnée » $(M S$, p. 121). En prenant ce point de vue, il est clair qu'on s'interdit toute possibilité, non seulement d'éviter l'erreur, mais encore d'en saisir la fonction positive, s'il est vrai que, comme le montre tout l'enseignement de G. Bachelard, la production des connaissances va de pair avec un apprentissage polémique qui trouve dans l'erreur, et notamment dans la recherche des sources de l'erreur, le moteur de son développement.

Une telle recherche est particulièrement nécessaire et urgente en sociologie, car, comme disent les auteurs, la sociologie est une science comme les autres qui rencontre néanmoins une difficulté spéciale à être une science comme les autres ( $M S$, p. 43). Il s'agit donc, tout d'abord, de mettre en lumière cette «difficulté spéciale »ou, ce qui revient au même, d'expliquer cette perméabilité particulière de la pratique sociologique à l'incidence de l'idéologie. A ce sujet, les remarques du Métier de sociologue apportent des éclaircissements décisifs.

En premier lieu, la sociologie, comme toute autre science, prête flanc aux attaques de l'idéologie à travers l'image que se font les chercheurs de leur propre 
pratique et de la connaissance scientifique en général. Cette conception correspond à ce que L. Althusser appelle la «philosophie spontanée des scientifiques» (P.S.S.) ${ }^{12}$. La P.S.S. constitue en fait $l^{\prime} \ll$ atmosphère », nécessairement contaminée d'idéologie, dans laquelle les scientifiques accomplissent les tâches prescrites par leur place à l'intérieur du procès de production des connaissances. Il convient de souligner, cependant, que le contenu de cette philosophie n'est ni cohérent ni systématique :

...le contenu de la P.S.S. est contradictoire. Cette contradiction existe entre deux éléments qu'on peut distinguer et identifier :

- un élément d'origine interne, directement issu de l'expérience de la pratique scientifique : convictions de caractère matérialiste, réaliste, objectiviste...13 ...Je propose d'appeler cet élément : l'élément intrascientifique, matérialiste : élément $\mathrm{n}^{\circ} 1$.

- un élément d'origine externe... issu des philosophies de la science existantes... : notions sur la science en général, la nature de l'activité scientifique, etc. Je propose d'appeler cet élément : l'élément extrascientifique, idéaliste : élément $\mathrm{n}^{\circ} 2^{14}$.

Or, en règle générale, la P.S.S. est caractérisée par la dominance de l'élément $n^{\circ} 2$ (idéaliste). Cette dominance s'explique facilement, si l'on tient compte du fait que, en dernier ressort, seul l'élément $n^{\circ} 1$ peut être dit «spontané » au sens strict; par contre, l'élément $\mathrm{n}^{\circ} 2$, l'élément proprement idéologique, est le produit d'une démarche réflexive qui, du moins en principe, aspire à la systématicité : d'où son rôle dominant au sein de la P.S.S., d'où aussi la résistance coriace qu'il offre à la critique épistémologique.

À noter en outre que cet élément idéologique peut assumer - et assume en fait - des formes différentes dans l'histoire de la production des connaissances, et que, à l'intérieur d'une conjoncture théorique donnée, il peut être différemment représenté dans chacune des pratiques scientifiques existantes. Ainsi, dans la sociologie actuelle - et en général dans les disciplines sociales et humaines c'est l'empirisme qui, sauf quelques rares exceptions, constitue l'obstacle épistémologique principal provenant de la P.S.S. Ces remarques d'Althusser coïncident point par point avec celles du Métier de sociologue : «...en sociologie, l'empirisme occupe, ici et maintenant, le sommet de la hiérarchie des dangers épistémologiques. » (MS, p. 103; c'est nous qui soulignons).

Or, on peut caractériser l'empirisme comme une variante spécifique d'une idéologie plus générale : celle que Macherey appelle l'«idéologie du donné ${ }^{15}$ 》, et qui, grosso modo, correspond à ce que Bachelard, dans la Formation de l'esprit scientifique, nomme 1 '« obstacle substantialiste ${ }^{16} »$. Le schéma invariant de cette idéologie se ramène à la postulation d'une spontanéité du réel, de sorte que c'est le réel lui-même qui détiendrait, comme ses propriétés immanentes, les lois de sa propre constitution. Les opérations de la recherche sont ainsi réduites

12. L. Althusser, Cours de philosophie pour scientifiques.

13. Par exemple: - Croyance en l'existence réelle de l'objet de la connaissance scientifique, croyance en l'existence, c'est-à-dire absence de mise en doute de la validité de la connaissance scientifique, croyance en l'existence de la méthode scientifique " (ibid).

14. Ibid.

15.P. Macherey, Cours de philosophie pour scientifique, 6e cours (ronéotypé).

16. Cf. G. Bachelard, la Formation de l'esprit scientifique, Paris, Vrin, 1965, chap. vi. 
à un travail d'enregistrement d'un donné préalable qui, soit sous la forme de l'évidence, soit sous celle de l'intimité, renferme en lui sa propre connaissance. Le discours scientifique n'est donc pas autre chose que la simple reprise, la simple répétition, de ce qui, dans le réel, se donne. Comme dit Macherey, l'« expression la plus simplifiée et la plus complète » de cette idéologie se laisse formuler dans ces termes : " connaître, c'est reproduire un réel donné ${ }^{17}$ ».

Ce «donné » est, pour la variante empiriste de l'idéologie substantialiste, le produit de l'expérience «sensible», «concrète», «matérielle». Il est clair pourtant qu'on reste dans les limites de cette idéologie en prenant comme point de départ, non pas le « réel » ainsi conçu, mais le «pensé » ou, comme dans un certain structuralisme, "les structures de l'esprit humain»: c'est le cas du formalisme. Cette idéologie ne joue pourtant en sociologie qu'un rôle subordonné : aujourd'hui, et jusqu'à nouvel ordre, c'est l'empirisme qui occupe la place dominante dans la philosophie spontanée des sociologues ${ }^{18}$.

Les formes sous lesquelles cet empirisme se manifeste dans la pratique sociologique sont sans doute aussi variées que possible. Mais c'est surtout au niveau de la construction de l'objet d'analyse qu'il produit ses effets les plus redoutables. $\mathrm{Si}$, pour ne faire appel qu'à des sciences sociales, la linguistique et l'ethnologie, sous des formes encore contaminées de présupposés empiristes ou formalistes, ont cependant réussi à produire des instruments conceptuels et méthodologiques permettant la "distinction entre l'objet réel, préconstruit par la perception, et l'objet de science, comme système de relations construites expressément $\gg(M S$, p. 60$)$, la sociologie, elle, malgré mainte déclaration d'intentions et quelques rares exceptions, continue pour l'essentiel à s'orienter à partir de critères empruntés à l'expérience naïve des acteurs. C'est donc à juste titre que, non sans ironie, Bourdieu, Chamboredon et Passeron, font allusion à ces sujets de recherche - qui prolifèrent parmi les sociologues débutants — du type « les loisirs des adolescents d'un grand ensemble de la banlieue-est de Paris». Ce n'est pas par hasard, en effet, que le sociologue, alors qu'il se fait une obligation impérative de se tenir le plus près du concret même - c'est l'obsession commune à tout empirisme -, n'aboutisse dans la plupart des cas qu'à la construction d'un objet hallucinatoire (un artefact selon le Métier de sociologue) dont le seul intérêt: est souvent de présenter, sous une forme abrégée, les principaux fantasmes idéologiques du chercheur. Enfin, la division bureaucratique du travail sociologique, les découpages «théoriques» qui ne sont d'habitude que la traduction savante de découpages administratifs ou scolaires, les conflits de frontière arti-

17.P. Macherey, op. cit. Soit dit en passant, on reconnaîtra dans cette formule le principe qui oriente la lecture herméneutique. Rien de plus logique : c'est que l'herméneutique est, très exactement, un empirisme de la lecture.

18. A l'exception peut-être de secteurs très limités, où grâce à l'influence de l'idéologie structuraliste (qu'il faut distinguer avec soin de la pratique de l'analyse structurale en linguistique et en ethnologie) l'empirisme cède sa place à la variante formaliste. La critique de ce formalisme n'est pas négligée dans le Métier de sociologue; cf. p. 75-76 : * Ainsi, la mêrne intention méthodologique de ne pas s'en tenir aux expressions conscientes, peut conduire à prêter à des constructions telles que l'analyse hiérarchique des opinions le pouvoir de remonter des déclarations... aux attitudes qui en sont le principe, c'est-à-dire de transmuer magiquement le conscient en inconscient, ou, par une démarche identique, mais qui échoue par des raisons inverses, d chercher la structure inconsciente du message de presse par une analyse structurale qui ne peut, dans le meilleur des cas, que redécouvrir a grands trais quelques vérités premières détenues en toute conscience par les producteurs du message. * (C'est nous qui soulignons.) 
ficiels qui en découlent, ne peuvent que renforcer l'emprise de l'idéologie empiriste sur chacune des opérations et des étapes de la recherche sociologique ( $M S$, p. 60).

Or, la P.S.S. (à dominante empiriste) n'est pas la seule voie d'accès de l'idéologie à l'intérieur du travail sociologique. Il en existe une deuxième, celle-ci spécifique de la sociologie, qui permet de comprendre cette difficulté spéciale de la recherche sociologique à conquérir son droit à l'existence théorique. En effet, en rapport avec la P.S.S., mais différente d'elle, il existe, chez tous les sociologues, - chez les «agents de production» de la connaissance sociologique - un ensemble complexe et hétérogène de notions, images, « représentations », croyances, concernant spécifiquement l'existence sociale et les problèmes sociaux des individus et des groupes. Cet ensemble constitue ce que Bourdieu, Chamboredon et Passeron appellent la «sociologie spontanée des sociologues». Certes, on pourrait peut-être objecter que les sociologues ne constituent pas une exception quant aux dangers supplémentaires qui menacent leur pratique de recherche, car tous les scientifiques possèdent une représentation préscientifique de l'objet de leur propre discipline : s'il y a bien une «sociologie spontanée des sociologues» (S.S.S.), il existe aussi une "biologie spontanée des biologistes», une "physique spontanée des physiciens», une «chimie spontanée», etc. Pourquoi donc, pourrait-on demander, les sociologues seraient-ils les seuls exposés à ce surplus d'idéologie?

Sans doute, aucun chercheur n'est à l'abri des risques idéologiques provenant de sa propre conception de l'objet de sa science; Bourdieu, Chamboredon et Passeron ont donc raison d'évoquer la formule de Bachelard, d'après laquelle «tout chimiste doit combattre en lui l'alchimiste» (MS, p. 49). Cependant, une différence subsiste entre la situation du chimiste ou du physicien, et celle du sociologue, par rapport à leurs «idéologies spontanées». Il se trouve en effet que la S.S.S. est dans un rapport étroit, non seulement, comme nous le verrons, avec la P.S.S., mais encore et surtout avec ce que Althusser appelle les « idéologies pratiques», à savoir ces formations idéologiques historiquement données (par exemple, l'idéologie religieuse, politique, économique, etc.) qui constituent des conditions d'existence de toute formation sociale ${ }^{19}$.

En dernier ressort, la S.S.S. n'est qu'un sous-produit spécifique de ces idéologies indispensables à la pratique sociale. C'est ce caractère indispensable qui distingue ces idéologies (et, par conséquent, la S.S.S.) de celles portant sur l'objet des autres sciences et, en particulier, des sciences dites « exactes et naturelles ». Ainsi, par exemple, au sujet de l'idéologie alchimique, Herbert écrit : « Certes, la symbolique du Grand Euvre a pu alimenter des idéologies politiques... mais elle n'était pas intrinsèquement nécessaire à la pratique politique, à l'intérieur du tout social : un monarque devait avoir sa cour de juristes et de prêtres, il pouvait avoir aussi un alchimiste ${ }^{20}$.

C'est pourquoi l'idéologie alchimique tombe en désuétude une fois que la chimie, après Lavoisier, s'est constituée comme science; sans doute, elle subsiste de façon fragmentaire et non organisée dans la tête des individus, mais elle reste

19. Voir note 5 .

20. T. Herbert, * Réflexions sur la situation théorique des sciences sociales et spécialement de la psychologie sociale », Cahiers pour l'analyse, nos $1-2$, p. 150. 
limitée et non essentielle. En revanche, la S.S.S., sous-produit d'une formation idéologique pratico-sociale, ne disparâtt pas avec la fondation d'une science des modes de production et des formations sociales. Celle-ci ne saurait en aucun cas prendre la place de celle-là; ainsi la S.S.S. constitue (et constituera toujours sans doute) un obstacle épistémologique spécifique de la sociologie. Il se peut même que, sous certaines conditions, dépendant en premier lieu de l'état et du développement des connaissances sociologiques dans un domaine donné, la S.S.S. prenue une forme réfléchie, systématique, et apparaisse comme «théorie 》 (dominante) du domaine en question. L'histoire de la sociologie montre bien que cette possibilité s'est effectivement réalisée à plusieurs reprises; que, souvent, la simple sublimation théorique d'une idéologie pratique a réussi à prendre littéralement la place de la théorie (ignorée ou refoulée) d'un domaine sociologique ${ }^{21}$.

Or, la S.S.S. admet elle aussi une pluralité de variantes - celles-ci ayant des rapports qui peuvent aller de la complexité ouverte jusqu'au conflit avec la P.S.S. Dans ce cas aussi, l'intervention épistémologique ne peut aspirer à être efficace qu'à condition de déceler préalablement les formations idéologiques qui jouent le rôle principal dans la conjoncture théorique. $\AA$ ce sujet, les analystes du Métier de sociologue montrent sans la moindre équivoque que c'est l'idéologie humaniste qui constitue, à l'heure actuelle, l'élément absolument dominant dans la S.S.S. : «La sociologie spontanée... doit sa cohérence psychologique au caractère systématique des illusions qu'elle procure... ( $M S$, p. 159).

On peut ajouter - et ce ne serait que prolonger la pensée des auteurs que si l'idéologie humaniste est aujourd'hui le thème central de la S.S.S., c'est parce que l'humanisme est une source inépuisable d'illusions ${ }^{22}$ :

La résistance que suscite la sociologie lorsqu'elle prétend déposséder l'expérience immédiate de son privilège gnoséologique s'inspire de la même philosophie humaniste de l'action humaine que certaine sociologie qui, en s'armant par exemple de concepts comme celui de «motivation » ou en s'attachant par prédilection aux questions de decision-making, réalise, à sa manière, le vou naïf de tout sujet social : entendant rester maître et possesseur de lui-même et de sa propre vérité, ne voulant connaître d'autre déterminisme que celui de ses propres déterminations (même s'il leur concède l'inconscience), l'humaniste naif qu'il y a en tout homme ressent comme une réduction «sociologiste » ou \& matérialiste » toute tentative pour établir que le sens des actions les plus personnelles et les plus «transparentes » n'appartient pas au sujet qui les accomplit mais au système complet des relations dans lesquelles et par lesquelles elles s'accomplissent ( $M S$, p. 39).

Enfin, l'humanisme, idéologie pratico-sociale, peut lui aussi assumer plusieurs formes : non seulement peut-il se dissimuler sous le langage «scientifique » des «besoins sociaux», des «motivations》 et des «prérequis fonctionnels》, mais encore peut-il s'articuler sur d'autres thèmes idéologiques provenant, soit des idéologies pratiques, soit de la P.S.S., soit encore des deux. L'humanisme d'inspiration religieuse est un exemple de la première possibilité, l'humanisme historiciste (greffé sur une conception empiriste de la connaissance et une idéologie vague de la «dynamique » ou du changement social) un exemple de la dernière.

21. La dominance, pendant plusieurs décennies, de l'idéologie * fonctionnaliste * en est un exemple, et non des moins significatifs.

22. Comme nous le verrons, la domination de l'humanisme en sociologie s'explique encore pour d'autres raisons. 
Sans doute, c'est cette deuxième variante qui joue le rôle d'obstacle idéologique principal dans la pratique sociologique actuelle, et pour cause.

En effet, en accordant en même temps leurs droits aux illusions des agentssupports de la production (S.S.S.), à la P.S.S. (empirisme) et à l'image plus ou moins bergsonienne qu'elle se fait du changement historique, l'idéologie humaniste historiciste accomplit à la perfection la tâche théorique et pratico-sociale qu'on exige d'elle : elle se plaît à promouvoir une conception de l'Homme selon laquelle de «support », il devient «metteur en scène » de sa propre existence, elle affirme sa volonté scientifique à travers un empirisme qui s'exprime et souvent se sublime dans une philosophie obsédée du " concret ${ }^{23} »$, elle échappe enfin au fixisme et au dogmatisme de l'humanisme théologique en affectant à la « nature humaine » ainsi conçue des attributs de l'historicité concrète. D'où notamment l'intérêt aussi passionné que suspect que provoquent chez les sociologues de toute souche, les œuvres de la période humaniste-feurbachienne de Marx. C'est en effet dans ces ouvrages qu'on trouve (surtout dans les célèbres Manuscrits de 44, tentative de conciliation de l'anthropologie feuerbachienne et de l'historicisme hégélien) une définition historiciste de l'« essence humaine » comme «travail», «praxis consciente et libre », qui s'accorde à merveille avec les préjugés et les intérêts de la S.S.S. et de la P.S.S. ${ }^{24}$.

C'est de la reconnaissance et de l'analyse du rôle capital que joue, à l'heure actuelle, la formation idéologique humaniste historiciste, que l'ouvrage de Bourdieu, Chamboredon et Passeron tire son incontestable efficacité critique. Cette analyse et cette critique restent sans doute exemplaires. Elles suffisent pour faire de ce texte un instrument indispensable pour la «formation de l'esprit sociologique ».

Mais ce n'est pas tout. Très prudemment, certes, les auteurs ébauchent, comme nous l'avons indiqué, une \&stratégie théorique » visant à asseoir la pratique sociologique sur des bases solides. On doit bien parler de «stratégie » car, du moins dans ce premier volume, les auteurs se gardent bien de proposer une nouvelle théorie sociologique (ou simplement d'en donner les principes). Bien entendu, ce «silence » n'est pas objectable : une analyse destinée à fournir les règles et les principes d'une surveillance épistémologique efficace des opérations de la recherche sociologique n'a pas à prendre la place de cette recherche même : il n'y est question que de dégager la ligne épistémologique juste permettant l'approche de questions qui restent et resteront sociologiques ${ }^{25}$. Et pourtant certains paragraphes du Métier de sociologue semblent vouloir aller beaucoup plus loin. Celui-ci, par exemple :

La question de l'affiliation d'une recherche sociologique à une théorie particulière du social, celle de Marx, de Weber ou de Durkheim par exemple, est toujours seconde par rapport à la question de l'appartenance de cette recherche à la science sociologique : le seul critère de cette appartenance réside en effet dans la mise en auvre des principes fondamentaux de la théorie de la connaissance sociologique qui, en tant que telle, ne sépare

23. Par exemple chez G. Gurvitch, dont C. Lévi-Strauss, au chap. xvr de l'Anthropologie structurale, fait une critique violente mais, pour l'essentiel, juste.

24. Cf. L. Althusser, « Marxisme et humanisme », Pour Marx, Paris, F. Maspero, 1965.

25. Cf. L. Althusser, Cours de philosophie pour scientifiques, et Lénine et la philosophie, Paris, F. Maspero, 1969. 
aucunement des auteurs que tout séparerait sur le terrain de la théorie du système social (MS, p. 11; c'est nous qui soulignons).

Ainsi donc, si nous comprenons bien les auteurs, ce qui définirait en propre la scientificité de la sociologie est à situer, non pas au niveau du système de concepts permettant l'appropriation cognitive des "faits sociaux», mais plutôt au niveau de l'épistémologie sociologique, au niveau donc de la discipline vouée à l'exercice du contrôle syntaxique et sémantique des opérations de la recherche. Or, outre qu'il y aurait beaucoup à dire au sujet de cette prétendue unanimité des sociologues (et notamment de ceux mentionnés par Bourdieu, Chamboredon et Passıron, à savoir Marx, Weber et Durkheim) ${ }^{26}$ sur le terrain même de la "théorie de la connaissance du social ", il nous semble qu'un tel déplacement du principe d'unité de la science sociologique implique une double erreur :

a) On oublie tout d'abord que la production des connaissances sociologiques est l'effet propre, non pas de la «théorie de la connaissance du social», mais de la théorie du social elle-même. Les faits sociaux ne sauraient en effet être connus que par la mise en œuvre de concepts et d'énoncés sociologiques (et non pas épistémologiques);

b) On accorde ensuite à la pratique épistémologique une portée théorique et un statut qu'elle ne saurait jamais revendiquer. Il convient en effet de rappeler que l'épistémologie n'est pas une science, qu'elle est distincte des sciences, même si elle ne se définit que par le rapport privilégié (d'extériorité et d'intériorité) qui la relie aux sciences ${ }^{27}$.

Les conséquences de ce double malentendu apparaissent clairement dans le paragraphe consacré au thème : "Théorie de la connaissance sociologique et théorie du système social $\gg(M S$, p. 53-55). En effet, dans ce texte, après avoir dénoncé la confusion entre «l'exigence insoutenable d'une théorie générale et universelle des formations sociales et l'exigence inéluctable d'une théorie de la connaissance sociologique $\gg(M S, p, 54)$ - confusion encouragée, d'après Bourdieu, Chamboredon et Passeron, par les doctrines sociologiques du XIX siècle - les auteurs cherchent à définir ce rapport spécifique qui unirait l'épistémologie sociologique (désignée avec la formule ambiguë de «théorie de la connaissance sociologique ») à la théorie du social proprement dite. Or, ce n'est certainement pas par hasard que, d'une part, ce lien soit réfléchi en faisant appel à la notion idéologique de «fondement » et que, d'autre part, il mette en rapport une épistémologie dite « unitaire», " principe générateur des actes et des discours sociologiques », et des théories sociologiques «partielles», «limitées à un ordre défini de faits » $(M S$, p. 54). En effet, c'est une seule et même décision théorique qui accorde à l'épistémologie sociologique le rôle de principe fondateur du savoir sociologique (renouvelant ainsi le vieux rêve de la philosophie comme «fondement: » des sciences) et qui morcelle l'objet de la sociologie en un ensemble indéfini de sous-objets partiels dont l'unité, «épistémologique», reste nécessairement extérieure aux systèmes conceptuels qui les définissent. Un tel point de vue n'est

26. On ne saurait en effet, malgré quelques convergences souvent réelles, parfois simplement verbales, tenir ensemble » l'épistémologie weberienne, inspirée dans une grande mesure du néo-kantisme allemand, c'est-à-dire d'une philosophie idéaliste de la science, et le matérialisme dialectique.

27. Cf. L. Althusser, Lénine et la philosophie, p. 35. Cf. aussi, A. Badiou \& Le reconmencement du matérialisme dialectique $*$, Critique, mai 1967, p. 445 sqq. 
d'ailleurs pas éloigné de celui de R. K. Merton, avec sa conception des «théories à moyenne portée » (à laquelle Bourdieu, Chamboredon et Passeron font explicitement référence), et reste donc susceptible des mêmes critiques.

En effet, si par «théorie universelle du système social» on entend un ensemble organisé de propositions sociologiques rendant compte de toutes les formations sociales réelles ou simplement virtuelles, il est clair que l'exigence d'un tel corpus théorique est insoutenable. $\mathrm{Si}$, par contre, on entend par « théorie du système social $\gg$ un système de concepts abstraits, dont la pertinence et la portée théoriques lui accordent le droit de constituer le point de départ nécessaire de toute analyse sociologique, cette exigence est non seulement légitime mais encore urgente. Mais ce système de concepts (définissant l'objet spécifique, relativement autonome, de la sociologie) reste par principe sociologique (même s'il peut et doit être soumis, lui aussi, à la surveillance épistémologique) ${ }^{28}$.

Certes, ce malentendu ne limite pas la valeur et l'intérêt des analyses de Bourdieu, Chamboredon et Passeron : il exige pourtant de poser la question du statut scientifique de la sociologie en d'autres termes que ceux des textes qu'on vient d'analyser. Autrement dit, si cette question nécessite, pour être posée en des termes rigoureux, le secours du matérialisme dialectique, il n'en est pas moins vrai - et non seulement la pertinence mais encore les difficultés des analyses du Métier de sociologue le prouvent - que c'est sur le terrain du matérialisme historique qu'elle pourra être effectivement résolue. Il faut donc « changer de terrain ».

\section{MATÉRIALISME HISTORIQUE ET SOCIOLOGIE}

... la tâche $\mathrm{n}^{\circ} 1$ de toute discipline nouvelle consiste à penser la différence spécifique de l'objet nouveau qu'elle découvre, à le distinguer rigoureusement de l'ancien objet, et à construire les concepts propres requis pour le penser. C'est dans ce travail théorique fondamental qu'une science nouvelle conquiert de haute lutte son droit effectif à l'autonomie ${ }^{29}$.

Ce texte d'Althusser définit rigoureusement, à notre avis, les priorités actuelles de la recherche théorique en sociologie. Donner à la sociologie sa place dans l'espace des sciences : telle est, sans aucun doute, l'urgence première que la conjoncture théorique et idéologique pose au travail sociologique. En fonction de cette urgence, une hiérarchie d'actes épistémologiques s'établit naturellement, une série ordonnée de tâches théoriques dont le point de départ consiste dans la mise en cuvre d'une réflexion visant la production des concepts majeurs de la pratique sociologique.

Or, s'il faut ici revenir à Marx, ce n'est pas seulement parce qu'il avait compris la nécessité et l'urgence de ce travail de construction conceptuelle, mais avant tout et surtout parce que son entreprise scientifique, dans son inachèvement

28. Ce malentendu transparaît dans les hésitations mêmes du texte; ainsi, selon les auteurs (p. 50), il serait question d'e opposer à l'image traditionnelle de la théorie une théorie proprement scientifique ou, d tout le moins, une théorie scientifique de la théorie scientifique $\star$. (C'est nous qui soulignons.) Cf. aussi p. 38, note 2.

29. L. Althusser, . L'objet du Capital *, dans Lire le Capital II, Paris, F. Maspero, 1965 , p. 125, note. 
même, indique déjà en creux cette place reconnue-méconnue par les discours sociologiques dominants. Que cette entreprise scientifique soit restée et reste encore inachevée, que la «reproduction méthodique de l'objet de la science des formations sociales ${ }^{30} »$, pour l'essentiel, n'ait pas eu lieu (seule exception : Lénine), s'explique par le prodigieux refoulement dont le matérialisme historique a été l'objet, refoulement inscrit nécessairement dans cette histoire du mode de production capitaliste que la science marxiste avait justement réussi à déchiffrer. Cependant, aujourd'hui que, grâce surtout aux analyses de Louis Althusser et de son équipe, la censure historique qui s'est exercée sur le discours marxiste commence laborieusement à être levée, on peut espérer que la reprise rigoureuse de ce discours rejoigne le centre même de l'interrogation sociologique. En ce sens, les résultats étonnants obtenus par Poulantzas dans Pouvoir politique et classes sociales montrent déjà que cet espoir n'est pas illusoire.

La pertinence et la richesse des analyses de Poulantzas ne sauraient sans doute être exagérées. Nous voudrions pourtant ici nous limiter à reprendre un seul point - cependant capital - de ces analyses : celui justement où, sous une forme encore largement implicite, s'opère la jonction de la problématique globale du matérialisme historique avec la question particulière de l'objet de la sociologie. Nous faisons évidemment allusion aux remarques que Poulantzas consacre au problème de la construction du concept de \&classe sociale " (cf. notamment chap. II, "Politique et classes sociales》).

On sait que la question du statut théorique des «classes sociales » supporte aujourd'hui le poids d'une lourde histoire. Cette histoire concerne sans doute la pensée marxiste, mais non seulement ni principalement du point de vue théorique au sens strict, mais plutôt des points de vue idéologique et politique. Dans Histoire et conscience de classe, G. Lukacs déplore le fait que les analyses du Capital s'arrêtent précisément au moment où Marx aborde le problème des classes sociales dans le mode de production capitaliste. Mais les raisons d'un tel regret ne sont que secondairement théoriques : face au mécanicisme réformiste de la $\mathrm{I}^{\mathrm{e}}$ Internationale, face à une théorie et à une pratique marxistes appauvries et sclérosées, la réflexion de Lukacs - comme d'ailleurs celles de Korsch et de Gramsci - visait à mettre en valeur la question des classes sociales en vue d'un objectif politique précis : il s'agissait de rétablir dans ses droits la praxis révolutionnaire, de redonner toute sa portée théorique, et surtout pratique, à la célèbre formule de Marx, d'après laquelle les «hommes concrets» - et non pas les forces mécaniques - sont les vrais acteurs et auteurs de l'histoire.

Bien entendu, il n'est pas question ici de porter un jugement sur la pertinence politique de cette réaction * gauchissante »; d'autres l'ont déjà fait et, en premier lieu Lénine - cf. la Maladie infantile du communisme (le gauchisme »). Si nous avons fait mention de Lukacs c'est plutôt pour le caractère tout à fait typique de sa démarche : il illustre de façon presque exemplaire l'invariant de toute problématique théorique portant sur la question des classes sociales, à savoir la mise en place d'un ensemble notionnel faisant de ces dernières le lieu d'émergence central, sinon exclusif, de l'efficacité historique. Qu'un tel ensemble

30. T. Herbert, « Pour une théorie générale des idéologies \$, Cahiers pour l'analyse, n॰ 9, p. 76. 
lié de notions aboutisse forcément à interpréter les classes sociales en termes de sujets collectifs, les remarques faites à propos de la logique de l'idéologie humaniste suffisent à en montrer la nécessité. On retrouve d'ailleurs cette constante théorique dans la plupart des «classiques » du marxisme (Lukacs, Korsch, Gramsci, R. Luxembourg, Labriola) ainsi que chez les non-marxistes (Weber, Dahrendorf, Gurvitch, Wright Mills et bien d'autres).

Ce qui semble, sinon surprenant, du moins symptomatique, c'est que cette contamination de la réflexion sur les classes sociales par une problématique idéologique de caractère humaniste apparaît même chez des intellectuels qui, comme Gramsci par exemple, ont donné les preuves d'une lucidité théorique exceptionnelle dans d'autres domaines de la pensée sociale. Tout se passe comme si, dans la question des classes, se condensaient tous les obstacles épistémologiques et idéologiques, tous les présupposés de la P.S.S. et de la S.S.S. dont on a fait l'analyse plus haut. Il y a sans doute lieu de penser qu'un tel phénomène n'est pas le produit du hasard.

Évidemment, l'incidence de l'idéologie humaniste à l'intérieur des cadres théoriques du matérialisme historique ne pouvait aboutir qu'à des contradictions et à des impasses. Il se trouve en effet que la logique propre de la démarche marxiste exclut par principe toute interprétation humaniste de la place et du rôle des agents de la production (et a fortiori des classes sociales). D'où un conflit qui, pour des raisons idéologiques et politiques, se résolvait d'habitude dans la pure et simple abdication des exigences théoriques de la problématique marxiste, cette abdication prenant généralement et pour les mêmes raisons, la forme d'un compromis : injection de Hegel (Lukacs), de Kant (austromarxisme), de Husserl (marxisme phénoménologique) ou de Piaget (marxisme génétique) dans le discours marxiste, qui se trouvait ainsi défini comme affecté d'un manque dont la suture justifiait et réclamait l'éclectisme, "non sans dégâts, écrit A. Badiou, la rigueur du discours marxiste étant en situation de jointure avec les parties effondrées et menant sa propre vie clandestine en dessous des parades nominales de la Révision ${ }^{31} »$.

Un des mérites incontestables de l'analyse de Poulantzas est d'avoir su mesurer le poids spécifique de cette histoire (et d'en avoir tiré les leçons nécessaires); aussi et surtout d'avoir, pour la première fois, pris au sérieux les exigences de rigueur inscrites dans la logique de la démarche marxiste; d'où le fait qu'elle tienne compte en même temps :

a) $\mathrm{Du}$ développement inégal du matérialisme historique, notamment en ce qui concerne la production des concepts portant sur les niveaux superstructuraux d'un mode de production et d'une formation sociale, sur les formes et les mécanismes d'efficacité d'une structure régionale sur ses éléments, et d'une structure sur une autre structure (théorie de la causalité structurale, esquissée par Althusser à partir des concepts de détermination, domination, indice d'efficace, surdétermination, etc.), sur les formes de transition d'un mode de production à un autre, enfin sur les modes de production autres que le mode de production capitaliste (féodal, asiatique, esclavagiste, socialiste, etc.); p. 439.

31. A. Badiou, «Le recommencement du matérialisme dialectique 》, Critique, mai 1967, 
b) Des prérequis théoriques que doit respecter la construction du concept de classe sociale, à savoir l'exclusion de toute perspective empiriste et humaniste, ce qui implique la mise en place d'un ensemble de concepts et d'énoncés théoriques tenant compte du caractère de «supports » des agents de la production, et l'élaboration des distinctions et des précisions nécessaires pour déterminer la nature et le rôle des classes (et des \& catégories » qui en dérivent : fractions, couches, strates, etc.) au niveau, soit d'un mode de production « pur », soit d'une formation sociale historiquement donnée. Un texte d'Althusser résume bien l'essentiel de ces exigences :

Pour penser la nature d'une classe sociale, il est indispensable de faire intervenir conjointement la détermination de la base économique, la détermination de la superstructure juridico-politique et la détermination de la superstructure idéologique. Il est également indispensable de faire intervenir le jeu intérieur à cette détermination conjointe, pour rendre compte des déplacements possibles de la domination entre ces différentes déterminations... [Les classes sociales] sont surdéterminées puisque, pour comprendre leur nature, il faut faire intervenir la causalité structurale des trois niveaux de la société, l'économique, le politique et l'idéologique - causalité structurale s'exerçant sous la forme d'une conjonction de ces trois déterminations structurales sur un même objet, et dans la variation de l'élément dominant au sein de cette conjonction ${ }^{32}$.

Sans prétendre aller au-delà de ce que ce texte autorise, et sans vouloir non plus dissimuler le caractère largement métaphorique de nos remarques, nous dirions que la difficulté spécifique que présente la construction du concept de clasise sociale tient au fait qu'elle exige de prendre en même temps (en les considérant comme des dimensions constitutives du même objet) le point de vue topique, le point de vue économique et le point de vue dynamique. Qu'il nous suffise de rappeler - pour limiter la portée de nos propos - que ces trois concepts (topique, économique et dynamique) appartiennent à un champ thécorique en principe étranger au matérialisme historique : celui de la psychanalyse.

La «topique » désigne en effet la « théorie ou point de vue qui suppose une différenciation de l'appareil psychique en un certain nombre de systèmes doués de caractères ou de fonctions différentes et disposés dans un certain ordre les uns par rapport aux autres, ce qui permet de les considérer métaphoriquement conme des lieux psychiques dont on peut donner une représentation figurée spatialement ${ }^{33}$ ». Transposé à l'analyse marxiste, le point de vue topique renvoie à la distinction des différents niveaux ou instances d'un mode de production ou d'une formation sociale, et à l'intérieur de chacune de ces structures régionales, à la détermination de la place relative de chaque élément dans le système (par exemple, au niveau de l'instance économique, les places relatives qui sont celles de la force de travail, du moyen et de lobjet du travail. Ajoutons que ceci est également valable pour les autres niveaux).

A son tour, le point de vue économique «qualifie tout ce qui se rapporte à l'hypothèse selon laquelle les processus psychiques consistent en la circulation

32. L. Althusser, cité par E. Terray, le Marxisme devant les sociétés a primitives *, Paris, F. Maspero, 1969, p. 139.

33. J. Laplanche et J.-B. Poutalis, Vocabulaire de la psychanalyse, sous la direction de D. Lagache, Paris, P.U.F., 1967, p. 484. 
et la répartition d'une énergie quantifiable (énergie pulsionnelle) c'est-à-dire susceptible d'augmentation, de diminution, d'équivalences ${ }^{34}{ }{ }^{3}$

On peut aussi sans peine «traduire» ce point de vue dans le langage du matérialisme historique : il serait alors question de rappeler le rôle des concepts de détermination, domination, indice d'efficace, surdétermination, condensation, déplacement, etc. (c'est-à-dire, les concepts rendant compte de la hiérarchie des efficaces combinées qui donnent forme à un mode de production ou à une formation sociale). Le point de vue économique renvoie notamment à la thèse de la détermination en dernière instance par la base économique et, sur le plan de l'analyse d'une structure régionale - celle de l'économie par exemple — à la thèse du rôle déterminant en dernière instance des moyens de production.

Or, il n'est pas inutile de souligner que ces deux points de vue sont constamment présents dans la démarche de l'analyse marxiste, quel que soit l'objet sur lequel elle porte. Cependant - et c'est ce qui fait la spécificité théorique du problème des classes sociales - pour la construction du concept de ces dernières, il est nécessaire d'incorporer aussi le point de vue que Freud appelle "dynamique », et qui «envisage les phénomènes psychiques comme résultant du conflit et de la composition de forces exerçant une certaine poussée, celle-ci étant en dernière instance d'origine pulsionnelle ${ }^{35} 》$. Sans doute est-il possible, dans ce cas aussi, de transposer le point de vue dynamique dans le champ théorique du matérialisme historique : il suffirait de remplacer, dans le texte, "phénomènes psychiques» par «processus historiques》 et «pulsionnelle 》 par " économique 》 - au sens marxiste du terme — pour reconnaître immédiatement le phénomène historique de la lutte de classes et pour retrouver les échos de la célèbre formule de Marx, d'après laquelle «la lutte de classes est le moteur de l'histoire ». C'est ici cependant que la difficulté à laquelle nous faisions allusion apparaît clairement; en effet, même en accordant une certaine pertinence aux remarques précédentes, il reste que l'incorporation du point de vue dynamique, indispensable à la théorie des classes sociales, semble mettre en question, du moins à première vue, les déterminations établies à partir de la topique et de l'économique. Car, si en fonction de ces deux points de vue, une certaine problématique est mise en place, qui conçoit le développement historique comme un «procès sans sujet», le point de vue dynamique, en mettant en valeur la scène conflictuelle des forces sociales, risque de réintroduire ce qu'il s'agissait justement de tenir à distance : une conception de l'histoire pour laquelle cette dernière apparaît comme l'entreprise singulière de ces sujets \& au deuxième degré » que seraient les classes sociales. En revanche, à éliminer purement et simplement la dynamique, on tombe dans le piège symétrique et inverse, à savoir, ignorer tout simplement le phénomène de la lutte des classes (et ses effets spécifiques au niveau d'un mode de production et d'une formation sociale), ce qui aboutirait en dernier ressort à rendre impraticables en même temps, et la théorie, et l'action politiques.

Or, il suffit de suivre de près l'exposé de Poulantzas pour s'apercevoir comment l'intelligence de ces difficultés oriente chacune des étapes de son argu-

34. J. Laplanche et J.-B. Poutalis, Vocabulaire de la psychanalyse, p. 125.

35. Ibid., p. 123. 
mentation. Ainsi, le commencement de droit de son analyse est la position des concepts majeurs de «mode de production 》 et de «formation sociale», position qui coïncide avec l'intégration des points de vue topique et économique :

a) Par «mode de production * on doit entendre « non pas ce que l'on indique en général comme l'économique, les rapports de production au sens strict, mais une combinaison spécifique de diverses structures et pratiques qui, dans leur combinaison, apparaissent comme autant d'instances ou niveaux, bref comme autant de structures régionales de ce mode... Le type d'unité qui caractérise un mode de production est celui d'un tout complexe $\grave{a}$ dominance, en dernière instance, de l'économique : dominance en dernière instance à laquelle on réservera le nom de "détermination» (PPCS, p. 10);

b) Par «formation sociale» on désignera en revanche « une unité complexe à dominante » produit de la combinaison articulée de, au moins, deux modes de production distincts (PPCS, p. 11).

Quelques éclaircissements sont ici indispensables. En premier lieu, il faut mettre l'accent sur le primat logique de la topique sur l'économique ce qui, soit dit en passant, rapproche à nouveau la démarche marxiste de celle de la psychanalyse freudienne ${ }^{\mathbf{3 6}}$ : ainsi, la position des instances ou structures régionales précède logiquement leur mise en rapport (et l'établissement des hiérarchies instance déterminante, instance dominante - qui en découle); en revanche, il n'en est pas moins vrai que l'économique ainsi conçue constitue une détermination essentielle des concepts de mode de production et de formation sociale, ainsi que des concepts rendant compte de chacune des structures régionales. Pour employer les termes d'Althusser : "La domination n'est pas un simple fait indifférent, elle est un fait essentiel à la complexité même. C'est pourquoi la complexité implique la domination comme essentielle à soi : elle est inscrite dans sa structu$\mathrm{re}^{37} . \gg$

En deuxième lieu, il convient de rappeler la distance théorique qui sépare les concepts de mode de production et de formation sociale (ce qui, d'ailleurs, confirme le primat de la topique) : \& Le mode de production constitue un objet abstrait-formel qui n'existe pas, au sens fort, dans la réalité. Les modes de production capitaliste, féodal, esclavagiste constituent également des objets abstraits-formels, car eux non plus ne possèdent pas cette existence. Seule existe en fait une formation sociale historiquement déterminée, c'est-à-dire un tout social - au sens le plus vaste - à un moment de son existence historique : la France de Louis Bonaparte, l'Angleterre de la révolution industrielle (PPCS, p. 11). Cette distance reste cependant théorique, la position d'un objet comme existant hic et nunc n'étant pas, pour le matérialisme historique, le simple enregistrement d'un «donné », mais l'effet du déploiement nécessaire de la théorie même ${ }^{38}$.

36. Cf. J. Laplanche et S. Leclaire : "L'inconscient : une étude psychanalytique >, dans l'Inconscient (VI ${ }^{\mathrm{e}}$ Colloque de Bonneval, sous la direction de Henri Ey), Paris, Desclée de Brouwer, 1965, p. 95-130.

37. L. Althusser, Pour Marx, p. 207.

38. Comme dit A. Badiou : II est impossible d'opposer un concept théorique de l'histioire à une histoire réelle définie par sa complexité - impureté empirique. Dans l'épistémologie marxiste, la complexité est construite selon les concepts de la théorie. = (Cité par $\mathrm{E}$. Terray, le Marxisme devant les sociétés * primitives », p. 43). 
Enfin, l'expression même de «mode de production» renvoie à une des thèses centrales du matérialisme historique, à savoir celle du primat absolu de la production dans la structure d'un mode de production et d'une formation sociale. C'est ce que montre, d'après E. Balibar, l'analyse que Marx fait de la reproduction simple :

L'analyse de la reproduction montre que ces moments (circulation et consommation) ne possèdent pas d'autonomie relative, pas des lois propres, mais qu'ils sont déterminés par celles de la production... Il apparaît ainsi que le mode de production capitaliste détermine le mode de circulation, de consommation et de distribution. Plus généralement l'analyse de la reproduction montre que tout monde de production détermine des modes de circulation, de distribution et de consommation comme autant de moments de son unité ${ }^{3 \theta}$.

Cette remarque est capitale, car elle commande jusqu'à la démarche théorique aboutissant à la construction du concept de classe sociale. En effet, si la production est première et déterminante, il faut considérer nécessairement la structure de tout procès de production (économique, politique et idéologique) pour essayer d'en dégager les éléments pertinents pour la construction de ce concept. Or, l'analyse du procès de travail (chap. vII du Livre I du Capital) permet de dénombrer trois éléments constitutifs à savoir, objet, moyen et force de travail, reliés selon deux systèmes de rapports : rapports techniques constituant la structure du procès de travail comme mode d'appropriation de la nature par les producteurs (niveau des forces productives), rapports sociaux déterminant la structure du même procès, considéré du point de vue de l'appropriation sociale du produit (niveau des rapports de production) ${ }^{40}$. Il convient de souligner que, dans les deux cas, les agents de la production (travailleurs et nontravailleurs) sont définis, dans la perspective marxiste, comme des supports de la structure du procès. Ce qui veut dire qu'ils y occupent une place déterminée par la structure du procès de production de chaque région du mode de production ou de la formation sociale, et en particulier, par le rôle dominant des moyens de production.

La première grande thèse de Poulantzas est, bien entendu, que la théorie des classes sociales concerne les agents de la production en tant que supports des structures régionales d'un mode de production et d'une formation sociale. Cette thèse est spécifiée à travers la définition suivante qui peut être considérée comme une première élucidation du concept de classe sociale :

... la classe sociale est un concept qui indique les effets de l'ensemble des structures, de la matrice d'un mode de production ou d'une formation sociale sur les agents qui en constituent les supports : ce concept indique... les effets de la structure globale sur le domaine des rapports sociaux (PPCS, p. 69).

Il faut retenir dans cette définition deux déterminations décisives :

a) D'une part, la nécessité de prendre en considération l'ensemble des niveaux ou instances de la structure globale pour rendre compte de la nature spécifique des classes sociales. $\grave{A}$ ce sujet - et contre la conception classique qui tend à

39. E. Balibar, Sur les concepts fondamentaux du matérialisme historique », dans Lire le Capital II, p. 268-269.

40. Ibid., p. 204 et s. 
définir la classe sociale au seul niveau de l'instance économique - Poulantzas insiste sur le fait qu'une classe ne peut être considérée comme classe distincte que lorsque la position qu'elle occupe à l'intérieur des rapports de production économique se manifeste sur les autres niveaux par une «présence spécifique 》:

On peut dire que cette présence existe lorsque la relation aux rapports de production, la place dans le procès de production, se réfléchit sur les autres niveaux par des effets pertinents... On désignera par «effets pertinents 》 le fait que la réflexion de la place dans le procès de production sur les autres niveaux, constitue un élément nouveau, qui ne peut être inséré dans le cadre typique que ces niveaux présenteraient sans cet élément ( $P P C S$, p. 82).

Le concept d's effet pertinent» permet ainsi de comprendre - contre l'avis de Marx lui-même - que les «paysans parcellaires » constituaient, dans la formation sociale française à l'époque du Second Empire, une classe sociale au sens fort du terme, car leur existence économique se manifestait aux niveaux politique et idéologique, par le phénomène historique du bonapartisme.

b) D'autre part, il faut retenir aussi la distinction, indiquée déjà dans la définition, entre le domaine des \& structures \& et le domaine des \& rapports sociaux ». Cette distinction joue aussi un rôle théorique capital : \&... si en fait une classe sociale se présente comme un effet d'un ensemble de structures, encore faut-il délimiter exactement le domaine particulier sur lequel les effets de cet ensemble se réfléchissent en prenant la forme de la classe sociale 》(PPCS, p. 65).

En précisant que les classes se définissent comme l'effet global des structures régionales dans le domaine des rapports sociaux Poulantzas met en lumière l'existence d'un décalage théorique inhérent aux concepts de mode de production et de formation sociale, décalage qui ouvre la voie non seulement à la possibilité d'une étude relativement autonome des classes sociales mais encore - ce qui est essentiel - à l'introduction du point de vue dynamique dans la problématique en question. En effet, le domaine des rapports sociaux désignent spécifiquement le lieu des pratiques de classe qui assument toujours nécessairement une forme conflictuelle :

Les rapports sociaux consistent en pratiques de classe, les classes sociales y étant situées dans des oppositions : les classes sociales ne peuvent être conçues que comme pratiques de classe, ces pratiques existant dans des oppositions qui, dans leur unité, constituent le champ de la lutte des classes (PPCS, p. 90).

C'est donc dans le champ ainsi conçu des pratiques de classe que le point de vue dynamique fait son apparition. L'insertion de ce point de vue apparaît ainsi décalée par rapport à celle de la topique et de l'économique. Ce décalage doit cependant être précisé : à ce sujet, il faut d'abord rappeler que le domaine des structures est déterminant par rapport à celui des pratiques de classe; ensuite que cette détermination des pratiques par les structures n'implique pourtant pas l'impossibilité d'une intervention spécifique des pratiques sur les structures. En fait, ce qui permet de tenir ensemble les trois points de vue, c'est le fait que les déterminations découlant du niveau des structures, fixent les limites de l'efficacité de la lutte de classes et, en particulier, d'intervention des pratiques de classe sur les structures. 
On pourrait peut-être illustrer cette démarche en rappelant l'analyse que Marx consacre à la question de la détermination de la longueur de la journée de travail en système capitaliste. D'une part les contraintes biologiques, d'autre part les lois de la structure du mode de production capitaliste fixent les limites, maximales et minimales respectivement, de l'extension de cette journée. Mais ni les unes ni les autres suffisent à en déterminer précisément la longueur exacte à un moment historique donné : «Entre deux droits égaux (celui du capitaliste et celui de l'ouvrier) qui décide ? La force (c'est-à-dire, la lutte économique des classes) 》(Capital, I, p. 231).

Ajoutons enfin que, sous certaines conditions particulières mais dépendantes en dernier ressort des déterminations des structures, les pratiques de classe peuvent intervenir de façon décisive à l'intérieur d'une formation sociale : "alors - comme dit Marx dans Contribution à la critique de l'économie politique s'ouvre une époque de révolution sociale ».

Arrêtons ici ces remarques. Sans doute, des questions nombreuses restent ouvertes, et notamment celle de la détermination - au niveau de la définition de classe sociale - du seuil de pertinence, question sur laquelle les indications de Poulantzas doivent nécessairement être précisées et développées. En outre, certaines formules, dues en partie au soin de préciser la différence entre structures et pratiques, peuvent parfois laisser croire qu'on a affaire à deux domaines effectivement distincts d'un mode de production ou d'une formation sociale, ce qui, à la limite, conduirait en fait à transmuer une différence théorique en clivage ontologique ${ }^{41}$. Mais nous ne pensons pas que l'analyse de Poulantzas conduit nécessairement à une telle conclusion.

\section{CONCLUSION}

Il est temps maintenant d'introduire le principe qui a commandé notre analyse, et dont les remarques faites à propos du Métier de sociologue et de Pouvoir politique et classes sociales tirent leur exacte signification. À savoir, que c'est le domaine ainsi conçu des « rapports sociaux» (rapports qui, dans les sociétés de classe, se réalisent nécessairement comme des rapports conflictuels entre classes sociales) qui constitue en droit l'objet propre d'une sociologie scientifique. $\AA$ cette conclusion nous amènent, non seulement les analyses théoriques de Bourdieu, Passeron, Chamboredon et de Poulantzas, mais encore - sous la modalité nécessairement indicative qui est le propre de l'idéologie - les formes typiques qu'assume à l'heure actuelle la pratique sociologique elle-même. Car, derrière les définitions à demi vagues, à demi tautologiques qui prolifèrent dans les discours sociologiques contemporains ( $\ll \mathrm{La}$ sociologie est l'étude de l'action sociale », «la sociologie est l'étude des groupes», etc.), derrière aussi l'empirisme et l'humanisme qui qualifient aussi bien les démarches théoriques que les recherches "concrètes 》, il est néanmoins possible de déceler la reconnaissance allusive de cet objet; au-delà de ses formulations diverses, la question des rapports sociaux reste en fait le lieu commun, indiqué et méconnu en même temps, de la

41. *...entre le concept de classe, connotant des rapports sociaux, et les concepts connotant des structures - écrit Poulantzas -, il n'y a pas d'homogénéité théorique * (ibid., p. 70). 
pratique sociologique. Certes, à ignorer ce qui, par principe, structure ce domaine des pratiques de classe, la voie reste ouverte au renversement idéologique qui fait, systématiquement prendre l'effet pour la cause et vice versa. Dans la théorie sociologique cette inversion des déterminations prend une forme spécifique invariante : prendre l'effet pour la cause, c'est, en sociologie, prendre les supports pour sujets. On comprend ainsi que l'humanisme ne soit pas un simple "corps étranger » dont la sociologie saura se débarrasser à peu de frais, mais, bien au contraire, la forme même de l'idéologie sociologique : le risque et la tentation permanentes qui menacent (et menaceront toujours) le travail de décryptage du texte social.

RÉSUMÉ

À partir d'une lecture critique de Metier de sociologue par P. Bourdieu, J.-C. Chamboredon et J.-C. Passeron, et de Pouvoir politique et classes sociales par N. Poulantzas, l'auteur analyse les rapports complexes entre la pratique sociologique, l'épistémologie marxiste et le matérialisme historique, ce dernier étant conçu comme science des modes de production et des formations sociales. Il s'agit de montrer la complémentarité nécessaire entre l'épistémologie et la réflexion théorique en vue de l'établissement de concepts de base visant à définir l'objet propre de la sociologie et à lui conférer ainsi son droit à l'existence théorique.

\section{ABSTRACT}

[Towards a Science of Social Context] Starting with a critical discussion of Métier de sociologue by P. Bourdieu, J.-C. Chamboredon and J.-C. Passeron, and of Pouvoir politique et classes sociales by $\mathbf{N}$. Poulantzas, the author analyses the complex relationship between sociological practice and marxist epistemology and historical materialism, this latter being conceived as the science of the means of production and social structure. The problem is to show the necessary complementarity between epistemological discipline and theoretical reflection with the objective of producing basic concepts to define the proper object of sociology and, in so doing, to give the sociology a right to theoretical status.

\section{RESUMEN}

[Hacia una ciencia del texto social] Sobre la base de una lectura crítica de Métier de sociologue de P. Bourdieu, J.-C. Chamboredon y J.-C. Passeron, y de Pouvoir politique et classes sociales de N. Poulantzas, el autor analiza las relaciones complejas que unen la práctica sociológica, la epistemología marxista y el materialismo histórico, este último concebido como ciencia de los modos de producción y de las formaciones sociales. Se trata de mostrar el carácter necesariamente complementario entre la vigilancia epistemológica y la reflexión teórica, con objeto de establecer conceptos de base destinados a definir el objeto propio de la sociología y, por consiguiente, a acordar a ésta última su derecho a la existencia teórica. 